\title{
A Technique for the Rapid Isolation of Single Bacterial Spores
}

\author{
By R. S. HOLDOM AND K. I. JOHNSTONE \\ Department of Bacteriology, University of Leeds
}

(Accepted for publication 22 September 1966)

\begin{abstract}
SUMMARY
Phase-bright bacterial spores are deposited singly and rapidly by means of a glass microloop at pre-marked sites on the surface of a cast block of agar gel. The block is then dissected and portions, each carrying a single spore, are transferred to fluid for heat-resistance or other tests. Under optimal conditions of culture $96-100 \%$ of these single spores germinated and gave visible growth.
\end{abstract}

\section{INTRODUCTION}

The technique was devised to enable heat-resistance tests to be made on single spores of Bacillus subtilis in batches of multiples of 24. One worker can select and isolate approximately 100 spores per hour and the isolates can be preserved at $3^{\circ}$ without loss of phase-brightness or viability (i.e. capability of germination and production of visible growth), for at least 9 weeks. Thus large batches of spores can be prepared and the distribution of heat resistance amongst them estimated, knowing that each spore is phase-bright. It was important that the spores tested should be of approximately the same age because the age of spores is known to affect their response to germinants and recovery media (Magoon, 1926; Curran \& Evans, 1947), to heat-shock treatment (Curran \& Evans, 1947; Lawrence, 1957; Krishna Murty, 1957) and to heat resistance tests (Church, Halvorson \& Bontempo, 1957; Rayman, 1957; Evans \& Curran, 1960).

\section{METHODS}

The agar block. New Zealand agar $(1 \cdot 75 \%$, w/v, in distilled water) was clarified with Hyflo-super-cel diatomaceous earth (Koch-Light Laboratories, Colnbrook, Buckinghamshire) to remove bacterial debris (Feinberg, 1956). Sterile blocks of the gel 2.0 $\mathrm{mm}$. thick were cast between microscope slides. Each block, mounted on a sterile slide, was marked with 24 pairs of pits which were visible to the unaided eye (Fig. 1), being the imprints of the tips of 48 sterile steel needles mounted in an enclosed punch. The zone between each pair of pits was the site for the deposition of a single spore and was in register with the readings of the mechanical stage of the microscope, being $3.0 \mathrm{~mm}$. distant from the adjacent sites in both directions. The agar blocks and their slides were protected from aerial contamination by enclosure in Petri dishes, each also containing a sterile duralumin humidifying trough $4 \mathrm{~cm} . \times 2 \mathrm{~cm}$. and $0.75 \mathrm{~cm}$. deep (internal dimensions) holding glass wool saturated with distilled water, to minimize desiccation of the gel. During inoculation only, each block was protected from contamination of the sterile surface from droplets scattered from the inoculation loop, by a sterile metal cover, except for the extreme end remote from the pre-marked sites. 
Preparation of spores. A single-spore strain of Bacillus subtilis CN788 (Wellcome Research Laboratories, Beckenham, Kent) designated CN788/1 was used. At $37^{\circ}$, $18 \mathrm{hr}$ cultures on nutrient agar (Oxoid) produced mostly vegetative forms; a spore crop was obtained by inoculation from the nutrient agar culture on the surface of Tryptone soya broth (Oxoid) diluted $1 / 20$ with distilled water and solidified with $2 \%$ (w/v) agar. After incubation for $18-24 \mathrm{hr}$ at $37^{\circ}, 95 \%$ of the organisms were as free spores. With B. subtilis strains NCTC 3610 and NCTC 6432 (National Collection of Type Cultures), the yield of free spores was much reduced. The spores were harvested in sterile Ringer's solution with $0.1 \%(\mathrm{w} / \mathrm{v})$ gelatin and were not washed. Inoculation
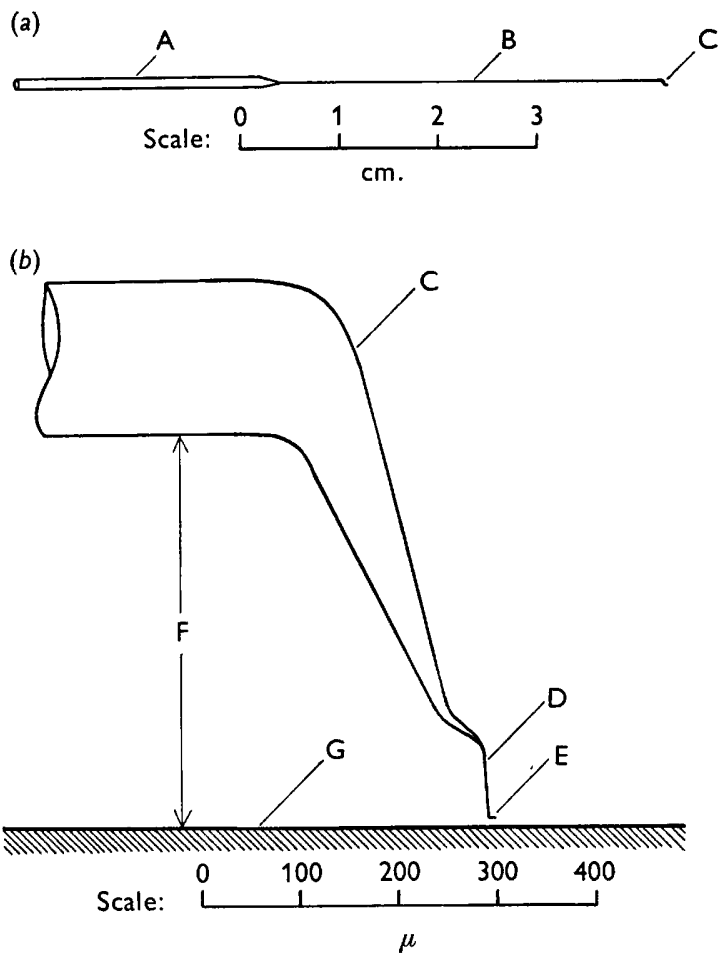

Fig. 1

Fig. 2

Fig. 1. Plan of a completed agar block showing the site of inoculation A, the section $B$ removed for sterility test and the 24 isolation sites, each carrying a single spore between the two locating pits. The broken lines indicate the knife cuts used to separate the isolation sites. Twice actual size.

Fig. 2. Elevation of a glass microloop: (a) showing the handle A, the shaft B and the tip C; (b) the tip $\mathrm{C}$ further enlarged, showing the filament $\mathrm{D}$ and the loop $\mathrm{E}$ in relation to the gel surface $\mathrm{G}$. The distance $\mathrm{F}$ is the clearance between the shaft and the gel.

was made from a faintly turbid spore suspension with a wire loop $1 \mathrm{~mm}$. in diameter along a strip A (Fig. 1) on the exposed upper surface of the gel. The fluid was rapidly absorbed, leaving the spores on the gel surface for selection and transfer.

The microloop. The soft glass microloop used consisted of a handle A (Fig. 2a) $2.5 \mathrm{~cm}$. long and $1.0 \mathrm{~mm}$. diameter, a shaft $B 3.8 \mathrm{~cm}$. long and $0.15 \mathrm{~mm}$. diameter, with a shaped tip C (Fig. $2 a, b$ ) terminating in a minute oval loop made in a high- 
power microforge. The latter, working at a magnification of $\times 400$, enabled the terminal glass filament $\mathrm{D}$ to be drawn out to $1 \mu$ diameter and the loop $\mathrm{E}$ to be formed at its end, orientated to lie in the plane of the gel surface. For pure strain isolations, for which this method is very suitable, the loop can be as small as $3.0 \mu \times 1.5 \mu$ internal dimensions, its size being adjusted to limit its carrying power to a single spore. For multiple isolations of spores of the same strain, however, a larger loop $14.0 \mu \times 8.0 \mu$ is suitable and will carry 12 spores of Bacillus subtilis. The filament thickness and loop dimensions were determined by trial for a given strain. The loop must pick up or discharge the spores readily on the gel surface and when correctly formed and orientated can be used to make several thousand isolations if protected from damage.

The clearance F (approximately $400 \mu$ ) ensured that the shaft did not come into contact with the gel surface G. The dimensions of the tip allowed adequate working distance for the microloop between the gel and the protecting coverglass, which was held $1.0 \mathrm{~mm}$. above the gel surface by a sterile Perspex cell enclosing the agar block and itself resting on the slide. The handle of the microloop was operated by the focusing movements of a second microscope (Johnstone, 1953), a conventional micromanipulator being unnecessary. The microloop was sterilized by immersion of the shaft and tip in chromic + sulphuric acid-cleaning solution, being carefully washed in sterile distilled water and then in $95 \%$ (v/v) ethanol in water before use.

Optical system. The $\times 40$ phase-contrast objective must have a working distance of at least $1.0 \mathrm{~mm}$. below the coverglass and an N.A. of at least 0.70 ; the substage condenser must have a working distance of at least $3.5 \mathrm{~mm}$. At $\times 750$ magnification, with a green filter (Wratten 58), the non-germinated spores appeared phase-bright, clearly defined and in sharp contrast to the phase-dark germinated spores and the vegetative forms. Catadioptric systems with long working distance can be used to simplify the manipulations, since the working distance below the coverglass can be greatly increased and condensation on the coverglass is then less troublesome, but only at the cost of loss in resolution and the necessary increase in illumination with the possibility of damage to the spores.

Manipulation of the spores. The margin of the inoculated strip nearest to the premarked sites was located and the microloop was centred to the optical axis whilst poised above the gel. On lowering the loop to encircle a selected spore and then raising it, the spore usually adhered firmly to the interior of the loop. Twelve selected spores were thus collected in the loop successively.

Whilst the loop was raised, the first pair of marking pits was located by operation of the mechanical stage controls and, from the reading of the scales and verniers of the stage, the positions of all the remaining sites were readily calculated. The charged loop was lowered to touch the gel surface gently between the two pits. At this point, the spores floated within the loop in the water exuded from the gel by the slight pressure of the glass. They were then readily discharged from the loop by vibration at the moment when the rising loop broke contact with the gel surface. This was effected by an electric bell mechanism mounted to impinge on the under side of the bench and controlled by a foot-switch and rheostat. A setting of the rheostat was found by trial which usually caused one spore to be left on the gel when the loop was raised during vibration. When more than one spore was ejected, these were clearly visible on the gel surface and any spores in excess were picked up with the loop, leaving an isolated spore on the first pre-marked site. 
The second site was then brought into position, by using the readings of the stage, and thus twelve successive isolations were made. The loop was then re-charged with a further twelve spores from the inoculated area, for the completion of the block. Each block of 24 isolations can be completed in less than $10 \mathrm{~min}$., re-sterilization of the loop being unnecessary for the same spore suspension.

Storage of the block. For Bacillus subtilis spores, it was necessary to store the isolates on the gel for at least $24 \mathrm{hr}$ before transfer to fluid medium when the maximum viability was to be obtained with untreated spores. Under these conditions, the viability (i.e. the proportion of spores germinating and giving visible growth in optimal medium) was $96-100 \%$, as compared with $83 \%$ when the blocks were dissected and transferred to medium immediately.

Dissection of the agar block. This was done under a stereoscopic microscope at $\times 5$ magnification, with protection against aerial contamination by $(a)$ a cabinet enclosing the stage with a plate-glass roof below the objectives; $(b)$ a sterile transparent plastic hood covering the agar block and supporting slide, with an aperture to give access to the sharp stainless steel dissecting knives held in the hand.

The site of the inoculum A (Fig. 1) was first separated and discarded. A section of the gel $B$ between the inoculum and the nearest isolation sites was then cut out and removed. This was cultured in Tryptone soya broth for 7 days at $37^{\circ}$ and was proved to be sterile if the isolations were to be accepted as valid. The 24 isolation sites were then separated by knife cuts, by using the locating pits as guides, and were transferred in turn with a sterile platinum scoop to individual tubes of fluid for the required tests.

\section{Accuracy of the method}

(1) It was shown microscopically that, after dissection of a pre-marked agar block and transfer of 24 isolation sites with their isolated spores to a second slide, all the 24 spores were present on the gel surface and each was situated between its locating pits. Therefore exudation of water from the agar during cutting of the gel with a sharp knife did not displace the spores on the surface.

(2) In control series of isolates, transferred without heating or other treatment to an optimal medium, all 24 isolates frequently yielded the expected growth and must therefore each have been transferred with the portion of agar carrying the pre-marked site.

(3) Spread of growth from the inoculum did not occur on the sterile gel surface, as shown by the sterility of the section $B$ (Fig. 1).

\section{Effects of deviation from the preferred technique}

The object of the method described was to prepare batches of single spores for heatresistance tests, the interpretation of which is greatly simplified when the control (i.e. unheated) series gave a consistently high percentage viability. With spores of Bacillus subtilis $\mathrm{CN} 788 / 1,96-100 \%$ of single spores germinated and gave visible growth in $24 \mathrm{hr}$ at $37^{\circ}$ in a carefully prepared Tryptone soya broth (Oxoid ingredients). Similar results were obtained with $B$. subtilis strains NCTC 3610 and NCTC6432, but with slightly greater delay in the appearance of growth. This optimal result was decreased by: (a) the use of a medium which was inferior for the strain, especially when overheated during sterilization; $(b)$ suspension or washing of the spores before isolation in distilled water or buffer solutions other than Ringer's solution containing $0.1 \%$ 
gelatin; (c) exposure of the isolated spores to distilled water; $(d)$ failure to store the isolates on the gel surface for at least $24 \mathrm{hr}$ before dissection and transfer to medium.

The interrelation of these four factors is complex. Storage of the spores as isolates on the gel at $3^{\circ}$ for $24 \mathrm{hr}$ greatly decreased the loss in viability and the delayed germination which might arise from factors $(a),(b)$ and $(c)$, alone or together. Such storage should therefore be a standard technique for $B$. subtilis spores.

The method, without storage on the gel, is applicable to clostridial spores and has been used to obtain multiple single-spore strains of Clostridium botulinum type E, for which $82 \%$ of isolates were viable when cultured in meat broth.

This work incorporates part of the doctoral thesis of one of us (R.S.H.), who was supported by a grant from the Medical Research Council. We are grateful to Professor C. L. Oakley, F.R.S., for his interest and helpful criticism at all times.

\section{REFERENCES}

Church, B. D., Halvorson, H. \& Bontempo, J. (1957). Alanine deaminase activity in spores of $B$. cereus var. terminalis. Bact. Proc. p. 116.

Curran, H. R. \& Evans, F. R. (1947). The viability of heat-activatable spores in nutrient and nonnutrient substrates as influenced by prestorage or poststorage heating and other factors. J. Bact. 53, 103.

Evans, F. R. \& CURRAN, H. R. (1960). Influence of preheating, pH, and holding temperature upon viability of bacterial spores stored for long periods in buffer substrates. $J$. Bact. 79, 361 .

FeInBerg, J. G. (1956). Agar clarification. Nature, Lond. 178, 1406.

JoHNSTONE, K. I. (1953). Micromanipulation on an agar surface for the isolation and cultivation of single organisms. J. gen. Microbiol. 9, 293.

Krishna Murty, G. G. (1957). Enzymes dormant in the intact spore. In Spores, Ed. by H. O. Halvorson. p. 105. Washington: Am. Inst. biol. Sci.

LAWRENCE, N. L. (1957). Enzymes active in the intact spore. In Spores, Ed. by H. O. Halvorson. p. 94. Washington: Am. Inst. biol. Sci.

Magoon, C. A. (1926). Studies upon bacterial spores. I. Thermal resistance as affected by age and environment. J. Bact. 11, 253.

RAYMAN, M. M. (1957). Discussion in: 'Effect of moisture activity on germination' by R. J. Beers. In Spores. Ed. by H. O. Halvorson. p. 51. Washington: Am. Inst. biol. Sci. 